\title{
Analysis of female drivers' ECG characteristics within the context of connected vehicles
}

\author{
Xiaoyuan Wang \\ College of Electromechanical Engineering, Qingdao University of Science and Technology, Qingdao, \\ China and Tsinghua University, Beijing, China \\ Yongqing Guo \\ School of Transportation and Vehicle Engineering, Shandong University of Technology, Zibo, \\ China and Tsinghua University, Beijing, China
}

Chen Chen

School of Transportation and Vehicle Engineering, Shandong University of Technology, Zibo, China; Tsinghua University, Beijing, China and School of Transportation and Vehicle Engineering, Shandong University of Technology, Zibo, China

Yuanyuan Xia

College of Electromechanical Engineering, Qingdao University of Science and Technology, Qingdao, China, and

$$
\text { Yaqi Liu }
$$

School of Transportation and Vehicle Engineering, Shandong University of Technology, Zibo, China

\begin{abstract}
Purpose - This study aims to analyze the differences of electrocardiograph (ECG) characteristics for female drivers in calm and anxious states during driving. Design/methodology/approach - The authors used various materials (e.g. visual materials, auditory materials and olfactory materials) to induce drivers' mood states (calm and anxious), and then conducted the real driving experiments and driving simulations to collect driver's ECG signal dynamic data. Physiological changes in ECG during the stimulus process were recorded using PSYLAB software. The paired T-test analysis was conducted to determine if there is a significant difference in driver's ECG characteristics between calm and anxious states during driving.

Findings - The results show significant differences in the characteristic parameters of female driver's ECG signals, including (average heart rate), (atrioventricular interval), (percentage of NN intervals $>50 \mathrm{~ms}$ ), (R wave average peak), (Root mean square of successive), (Q wave average peak) and ( $S$ wave average peak), in time domain, frequency domain and waveform in emotional states of calmness and anxiety.

Practical implications - Findings of this work show that ECG can be used to identify driver's anxious and calm states during driving. It can be used for the development of personalized driver assistance system and driver warning system.

Originality/value - Only a few attempts have been made on the influence of human emotions on physiological signals in the transportation field. Hence, there is a need for transport scholars to begin to identify driver's ECG characteristics under different emotional states. This study will analyze the differences of ECG characteristics for female drivers in calm and anxious states during driving to provide a theoretical basis for developing the intelligent and connected vehicles.
\end{abstract}

Keywords Driving anxiety, Driving experiment, ECG signal characteristics, Female driver, Paired T- test

Paper type Research paper

\section{Introduction}

With the rapid development of transportation industry, the number of private cars increases dramatically, and the injury and deaths due to traffic accidents continue to increase annually. According to statistics, more than 90 per cent of traffic accidents are due to human factors, either directly or indirectly, 70 per cent of which are caused by vehicle drivers (Wang et al., 2013; Jia et al., 2006). To reduce the human impact on traffic safety, traffic scholars have come up with one

The current issue and full text archive of this journal is available on Emerald Insight at: www.emeraldinsight.com/2399-9802.htm

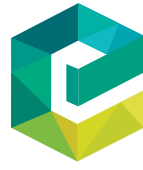

Journal of Intelligent and Connected Vehicles

Emerald Publishing Limited [ISSN 2399-9802] [DOI 10.1108/JICV-01-2019-0002]
(C) Xiaoyuan Wang, Yongqing Guo, Chen Chen, Yuanyuan Xia and Yaqi Liu. Published in Fournal of Intelligent and Connected Vehicles. Published by Emerald Publishing Limited. This article is published under the Creative Commons Attribution (CC BY 4.0) licence. Anyone may reproduce, distribute, translate and create derivative works of this article (for both commercial \& non-commercial purposes), subject to full attribution to the original publication and authors. The full terms of this licence may be seen at http://creativecommons.org/licences/by/4.0/legalcode

This study was supported by the Joint Laboratory for Internet of Vehicles, Ministry of Education - China Mobile Communications Corporation under Project [Grant No. ICV-KF2018-03], Qingdao Top Talent Program of Entrepreneurship and Innovation (Grant No. 19-3-2-8-zhc), the National Natural Science Foundation of China (Grant Nos. 71901134, 61074140, 61573009, 51508315), the Natural Science Foundation of Shandong Province (Grant No. ZR2017LF015).

Received 9 January 2019

Revised 29 March 2019

12 June 2019

14 August 2019

Accepted 24 October 2019 
Xiaoyuan Wang et al.

solution - cooperative driving (Chen et al., 2017). Cooperative driving with the connected vehicles is regarded as a promising driving pattern to significantly improve traffic safety (Jia and Ngoduy, 2016). Therefore, it is essential to promote the connected vehicles based on drivers' psychological and physiological characteristics, especially for the development of the intelligent human-machine interaction, personalized driver assistance system and driver warning system.

Yan et al. (2013) used the correlation analysis to determine the differences in driving anger among different types of vehicle drivers. The study found that during driving, male drivers are more likely to be angry than female ones, young drivers are more likely to be angry than elderly ones, and the average level of driving anger gradually decreases with an increase of driving experience. Schmidt-Daffy (2012) and Naveteur et al. (2013) proposed the target conflict model to analyze the influence of road visibility and time pressure on driver's anxiety. Results showed that the lower the visibility and the less the available time, the more anxious the drivers are. Geethanjali et al. (2018) used ANOVA (analysis of variance) to identify the impact of sound characteristics on driving emotion. It was found that driver's positive emotions tend to be induced by pleasant and low arousal sound, as well as the negative emotions tend to be induced by noisy and high arousal sound. Zhang et al. (2016) and Wang et al. (2016) used the K-mean clustering method to study the influence of environmental brightness on human emotion. Results showed that people's positive emotions are easily generated by high brightness of surrounding environment, and people's negative emotions are easily generated by low brightness of the environment. Dubovski et al. (2017) analyzed the influence of taste on human emotion using questionnaire survey and correlation analysis. The results revealed that anxiety is easy to be induced by bitterness or sour taste, and pleasure is easy to be induced by sweet taste. Jaeger et al. (2017) analyzed the correlation between young people's snack consumption choices and their emotions. It was found that for young people aged 18-25 years, their demands for spicy snacks and single snack intake increase significantly in anxiety compared with their states in calm. Kirwan et al. (2017) analyzed the relationship between insomnia and anxiety symptom severity. Results showed that the insomnia severity is positively correlated with the degree of anxiety. Researchers have also conducted the studies of the interaction between human emotion and physiological behavior in the fields such as family income (Clingingsmith, 2016), cultural difference (Ford and Mauss, 2015), violent crime (Vingilis et al., 2016), sport health (Kang and Gross, 2016), shopping consumption (Gallagher et al., 2017) and application of science and technology (Chen et al., 2017).

Emotion is not only a psychological activity, but it also closely related to physiological senses such as hearing, vision, taste and illness. Human emotions can cause peripheral physiological changes through autonomic nervous responses, such as electrocardiogram (Minhad et al., 2017; Reimer et al., 2011), respiration (Wilhelm et al., 2017; Velez et al., 2016), pulse (Handouzi et al., 2014), skin electricity (Goshvarpour and Abbasi, 2017; Barnard and Chapman, 2018), electromyogram (Rymarcayk et al., 2011) and skin temperature (Scott-Parker, 2017). Attention has been paid in the effect of human emotion on the changes of electrocardiograph (ECG) signal characteristics. Ba et al. (2016) used the wavelet transform feature extraction and BP (back propagation) neural network algorithm to study the changes of human skin electricity, skin temperature, heart rate and respiratory rate in positive and negative emotions. Results revealed that negative emotions present greater skin resistance, higher skin temperature, heart rate and breathing rate, than positive

Figure 1 Parts of the anxiety induction material

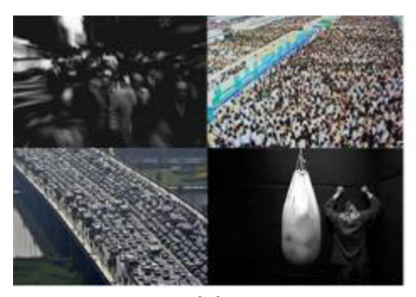

(a)

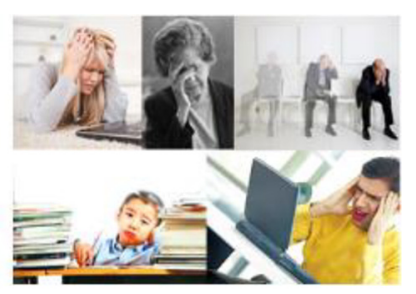

(b)
Notes: (a) Anxiety-induction visual materials; (b) pictures of different people in anxiety

Figure 2 Real vehicle driving experimental route

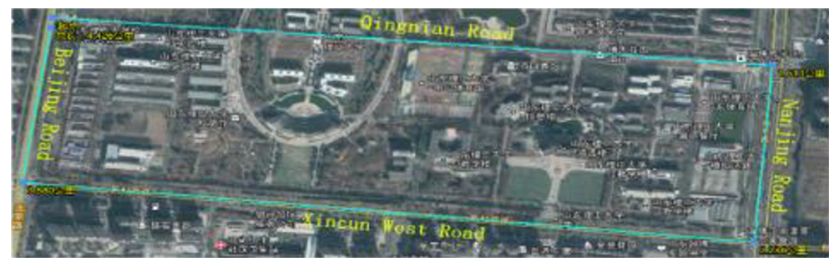

Figure 3 Real vehicle driving experimental equipment

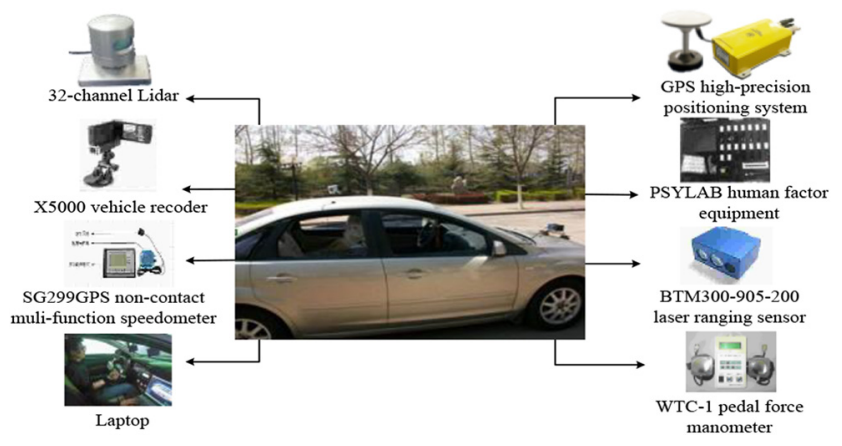

Figure 4 Screenshots of the experimental scenes (in Xincun West Road)

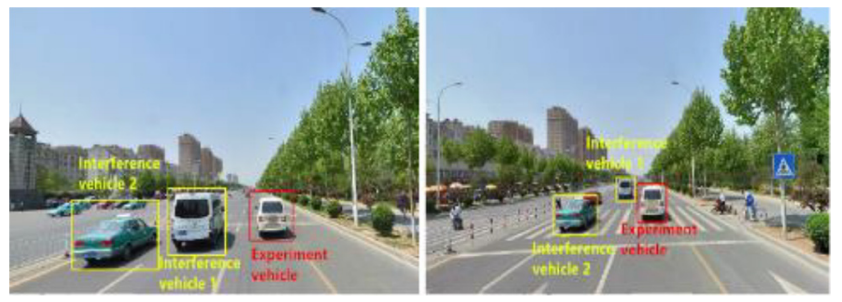




\section{Xiaoyuan Wang et al.}

Figure 5 Virtual driving experimental equipment

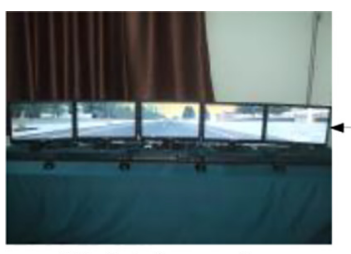

Workstation monitor

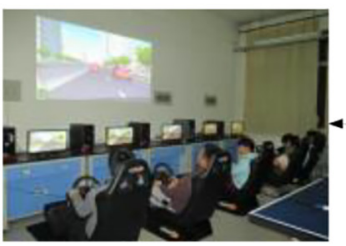

Muti-person muti-machine interactive virtual driving experiment platform

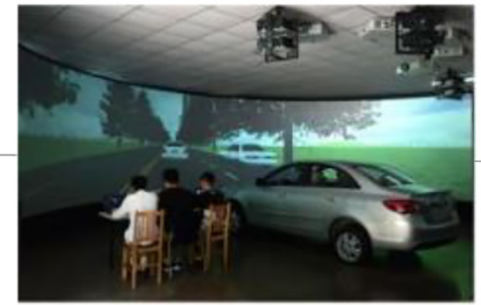

Virtual driving experiment scene

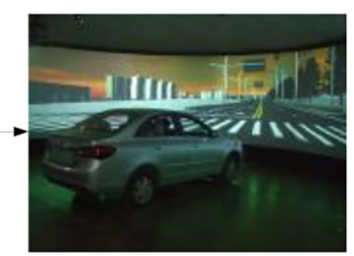

Virtual driving route

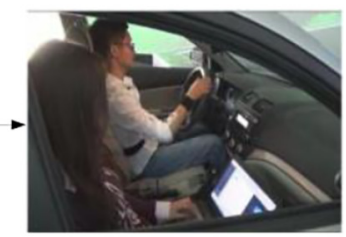

In-vehicle experimenter
Figure 6 The wearable wireless ECG sensors
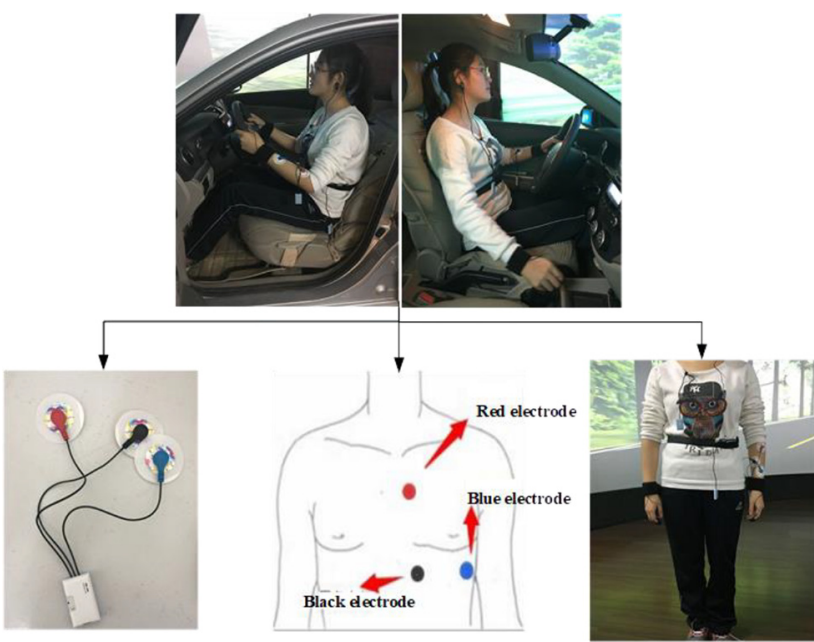

emotions. Takahashi et al. (2017) used the feature extraction and correlation analysis method to analyze the changes of human ECG signal in time, frequency and domain indicators in anger and calm states. It was found that the heartbeat interval is shorter in anger than in calm, and the ratio of low frequency band to high frequency band is greater in anger than in calm. Fernández (2016) analyzed the relationship between ECG waveform and behavioral difference using linear discriminant algorithm. Results showed that the QT interval variability of ECG waveform is positively correlated with human age and the degree of anger, while the RR interval variability of ECG
Figure 7 The simulation-based experiment route and street view
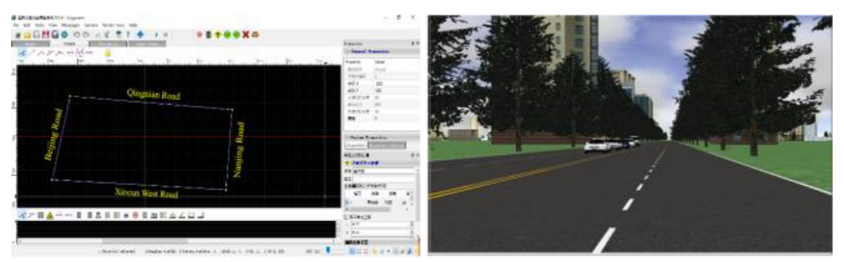

(a)
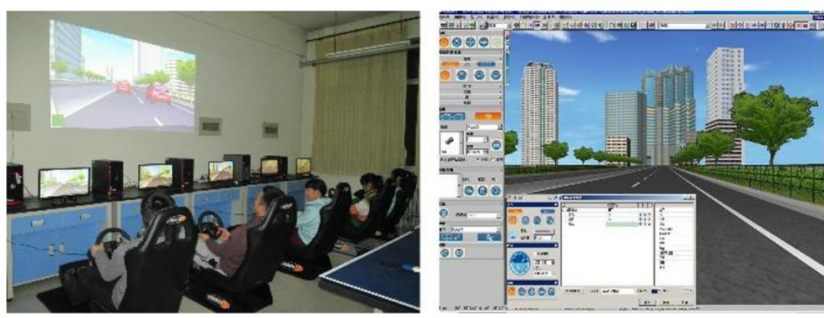

(b)

Notes: (a) Road Builder software interface and driving scene;

(b) Interactive Driving and UC-win/Road software interface

waveform is negatively correlated with human age and the degree of anger.

In conclusion, much research had been conducted to explore the influence factors for human emotion and the ECG signal characteristics of different emotions. However, only a few attempts have been made on the influence of human emotions on physiological signals in the transportation field. Hence, there is a need for transport scholars to begin to identify driver's ECG characteristics under different emotional states. This study will

Table I Technical specifications of ECG sensors

\begin{tabular}{lclc}
\hline Channel no. & 1 & Sample rate & $512 \mathrm{~Hz}$ \\
Measure range & $-6 \mathrm{mV} \sim+6 \mathrm{mV}$ & Noise & $1.6 \mu \mathrm{V}(\mathrm{RMS})$ \\
Accuracy & $0.183 \mu \mathrm{V}$ & Wave filter & $0.5 \mathrm{~Hz} \sim 45 \mathrm{~Hz}$ \\
Magnification & 500 & Common mode rejection ratio (CMRR) & $110 \mathrm{~dB}$ \\
\hline
\end{tabular}


Table II Hardware \& equipment of driving simulators

\begin{tabular}{|c|c|}
\hline System & Components \\
\hline Test vehicle & $\begin{array}{l}\text { Seat, acceleration pedal, brake pedal, clutch pedal, gear } \\
\text { lever, parking brake handle, steering wheel, instrument } \\
\text { panel, rearview mirror, etc. (The experimental vehicle is } \\
\text { reformed from a real vehicle.) }\end{array}$ \\
\hline $\begin{array}{l}\text { Visual display } \\
\text { system }\end{array}$ & $\begin{array}{l}\text { Desktop display, projection screen and rearview mirror } \\
\text { display }\end{array}$ \\
\hline Sound system & Stereo loudspeaker box \\
\hline Data collector & Data acquisition box \\
\hline
\end{tabular}

analyze the differences of ECG characteristics for female drivers in calm and anxious states during driving to provide a theoretical basis for developing the intelligent and connected vehicles.

\section{Research method}

\subsection{Participants}

In this study, a total of 18 female drivers were recruited and selected (age range $=22-28$ years; mean age $=24.6$ years) . Compared to men, women are more likely to experience anxiety (Panayiotou et al., 2017; Asher et al., 2017) and get involved in road traffic accidents (Hosking et al., 2010; Cestac et al., 2011). Subject's driving propensity was determined by driving propensity questionnaire (Wang et al., 2013), and all the subjects were categorized into extroverted drivers. In this study, if a subject drove more than 10,000 kilometers, she would be defined as an experienced driver, and a novice driver otherwise (Zhang, 2014). All the participants drove less than 10,000, whose average mileage was about $6,700 \mathrm{~km}$. Therefore, all the participants were classified into novice drivers. All the
Table III Beck anxiety inventory (BAI)

\begin{aligned} \hline 1 & Body numbness or thorns \\ 2 & Feel feverish \\ 3 & Leg tremble \\ 4 & Can't relax \\ 5 & Fear of bad things \\ 6 & Feel dizzy \\ 7 & Palpitation \\ 8 & Restless \\ 9 & Frightened \\ 10 & Tension \\ 11 & Suffocation \\ 12 & Hand trembling \\ 13 & Body shake \\ 14 & Afraid of out of control \\ 15 & Difficult breathing \\ 16 & Fear to die \\ 17 & Feel panic \\ 18 & Abdominal discomfort \\ 19 & Faint \\ 20 & Flush \\ 21 & Sweat \end{aligned}

Notes: The 21 symptoms in Table I have four levels of induction. The score of each symptom can be expressed as "1 point" means "none"; "2 points" means "mild, no major annoyance"; "3 points" means "moderate, feel uncomfortable but still tolerable"; "4 points" means "heavy, can only barely endure". The total score of 21 symptoms is $15-25$ points for mild anxiety, 2635 points for moderate anxiety and more than 36 points for severe anxiety

subjects were healthy and had no history of emotional psychosis, cardiovascular and cerebrovascular diseases. Before the experiment, they did not take any drugs that affect the nervous system within one week, and did not take

Figure 8 The experimental process of real driving and driving simulations in calm

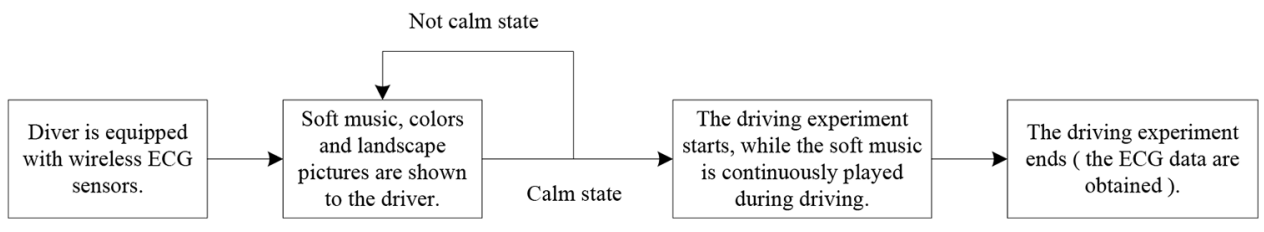

Figure 9 The experimental process of real driving and driving simulations in anxiety

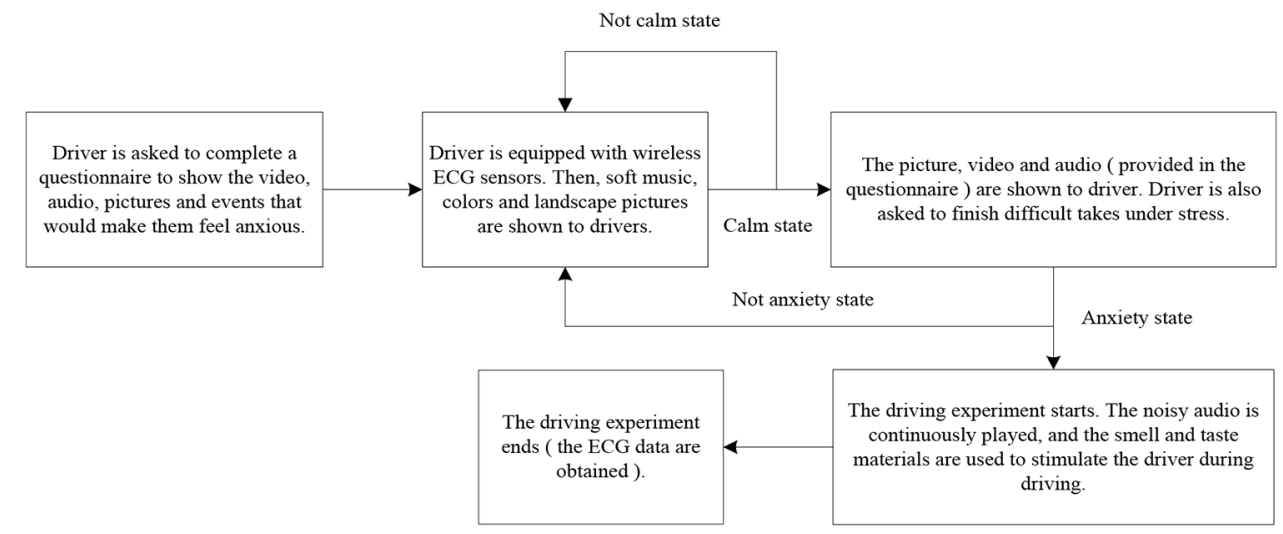


the foods and beverages (e.g. tea, coffee and wine) that affect the human mental state within $48 \mathrm{~h}$. In addition, they did not do any intense exercise and had a good rest. Prior to the experiment, researchers introduced the experimental environment and procedure to the participants in detail.

\subsection{Experimental material and equipment}

\subsubsection{Emotional induction materials}

The International Affective Picture System (IAPS) and the Chinese Affective Picture System (CAPS) were used as emotional induction materials. IAPS is an emotional material which is generally acknowledged around the world, and CAPS is an emotional material that adapts to the social and cultural context of China. Various anxiety-inducing materials were used in the experiments, including visual materials (e.g. words and pictures, brightness changes in driving environment), auditory materials (e.g. noisy and irregular sounds), multi-channel materials (e.g. videos and movies), olfactory materials (e.g. cigarettes and durian) and taste materials (e.g. balsam gourd and licorice). In addition, participants were also asked to finish difficult tasks under stress; as a result, their anxiousness was induced. For example, subjects were asked to complete a mobile game or solve math problems in a limited time. They would receive unexpected punishment if they failed to do. Parts of the anxiety-induction material are shown in Figure 1.

\subsubsection{Real driving experiment}

In the real driving experiments, the selected route starts from the west gate of Shandong University of Technology, passes through Qingnian Road, Beijing Road, Xincun West Road and Nanjing Road, and ends at the West Gate (as shown in Figure 2, the total length of $4.426 \mathrm{~km}$ ). The experiments were conducted in dry weather. In addition, an unmanned aerial vehicle (UAV) was used for recording the experimental process. The experimental equipment includes a comprehensive experimental vehicle, jamming vehicle, 32channel Lidar, BTM300-905-200 laser ranging sensor, GPS high-precision positioning system, SG299GPS non-contact multi-function speedometer, X5000 vehicle recorder, PSYLAB human factor equipment, WTC-1 pedal force

Figure 10 Denoising preprocess interface for original ECG signal

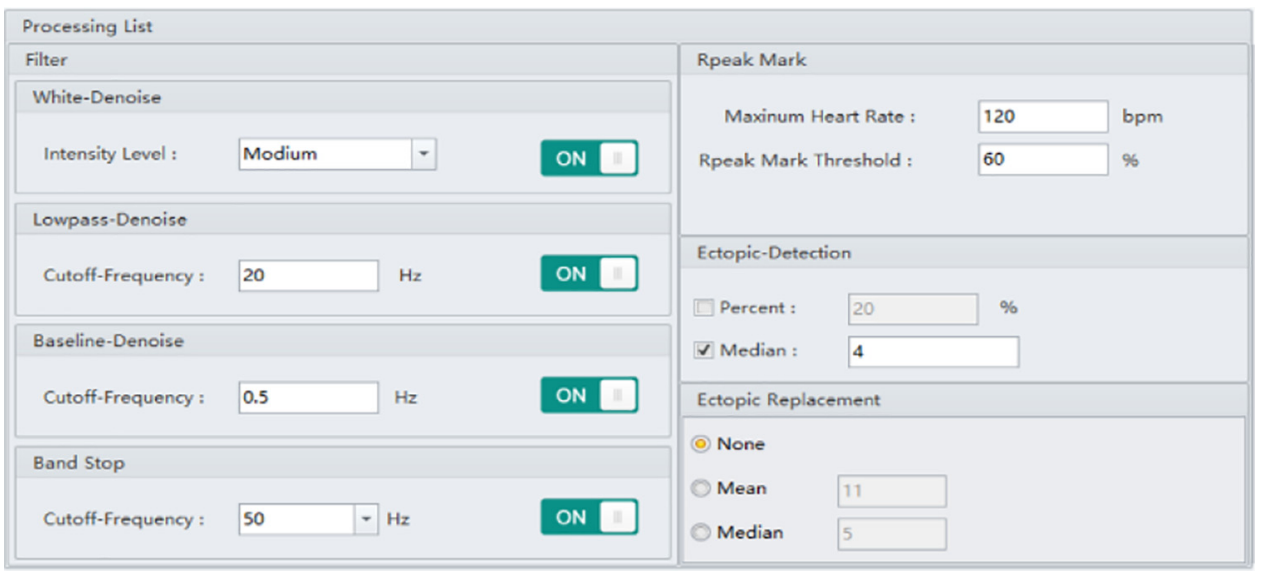

Table IV The definitions of parameters for denoising preprocess

\begin{tabular}{llll}
\hline White-Denoise & Baseline-Denoise & Lowpass-Denoise & Band stop \\
\hline Remove white noise from ECG signals & $\begin{array}{l}\text { High frequency signal is retained } \\
\text { and low frequency signal is cut off }\end{array}$ & $\begin{array}{l}\text { Low frequency signal is retained } \\
\text { and high frequency signal is cut off }\end{array}$ & Remove power frequency interference \\
\hline
\end{tabular}

Figure 11 Comparison of ECG signal before and after denoising

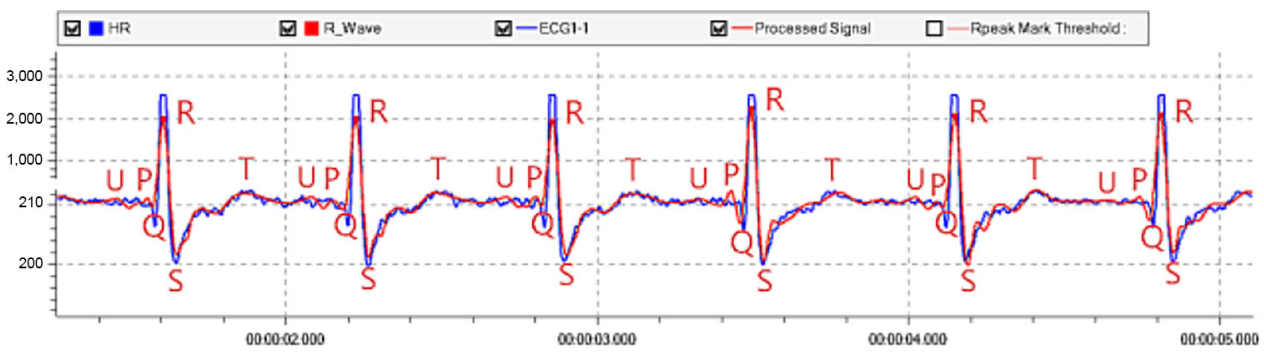


manometer, high-definition camera, laptop and UAV. Parts of the experimental equipment are shown in Figure 3. Screenshots of the real experimental scenes (in Xincun West Road) are illustrated in Figure 4.

Table V Variables and symbols for ECG signals

\begin{tabular}{|c|c|}
\hline Variable & Symbol \\
\hline Emotion & $E m$ \\
\hline $\mathrm{R}$ wave average peak (uV) & RWAVE \\
\hline T wave average peak (uV) & TWAVE \\
\hline Q wave average peak (uV) & $Q$ \\
\hline S wave average peak (uV) & $S$ \\
\hline Average heart rate $(b p m)$ & $A V H R$ \\
\hline Atrioventricular Interval ( $m s$ ) & AVNN \\
\hline $\begin{array}{l}\text { Standard deviation of NN intervals for period of } \\
\text { interest }(m s)\end{array}$ & SDNN \\
\hline Percent of NN intervals $>50 \mathrm{~ms}(\%)$ & $P N N_{50}$ \\
\hline Root mean square of successive ( $m s)$ & RMSSD \\
\hline $\begin{array}{l}\text { Ratio of ultra-low frequency band to very low } \\
\text { frequency band }\end{array}$ & UVLFIVLF \\
\hline Ratio of low frequency to high frequency band & IFIHF \\
\hline Total power $\left(m s^{2}\right)$ & $T P$ \\
\hline
\end{tabular}

\subsubsection{Driving simulations}

Using real driving experiments to collect data is timeconsuming, expensive and difficult to organize. It is difficult to obtain a large amount of real driving experimental data. Driving simulation was used as a supplement to real vehicle experiment because it is safe, low-cost and easy to control. The Road Builder and UC-win/Road software were used to construct the simulation-based experiment platforms of the human-vehicleenvironment comprehensive road system and the multi-person multi-machine interactive environment, based on the road attributes, traffic volume and other parameters of the field driving experiments. The virtual driving experiment equipment are shown in Figure 5. The wearable wireless ECG sensors are shown in Figure 6. The simulation-based experiment route and street view are shown in Figure 7. Technical specifications of ECG sensors are shown in Table I. Hardware \& Equipment of driving simulators are shown in Table II.

\subsection{Experimental process}

Considering the participants with insufficient driving experience, busy and complex traffic situations easily result in their emotional fluctuations. Therefore, the weekends from 6:00 a.m. to 8:00 a. $\mathrm{m}$. (with low traffic) were selected to conduct the real driving experiments in calm, to ensure the participants in calm while driving. The experimental process is shown in Figure 8.

Figure 12 Subjects' ECG data during driving in calm and anxiety

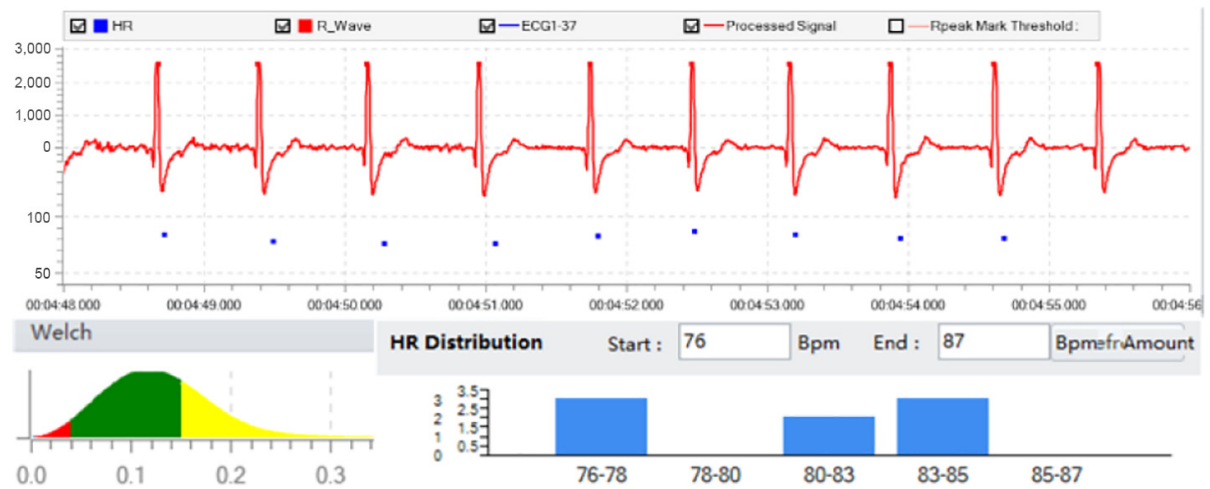

(a)

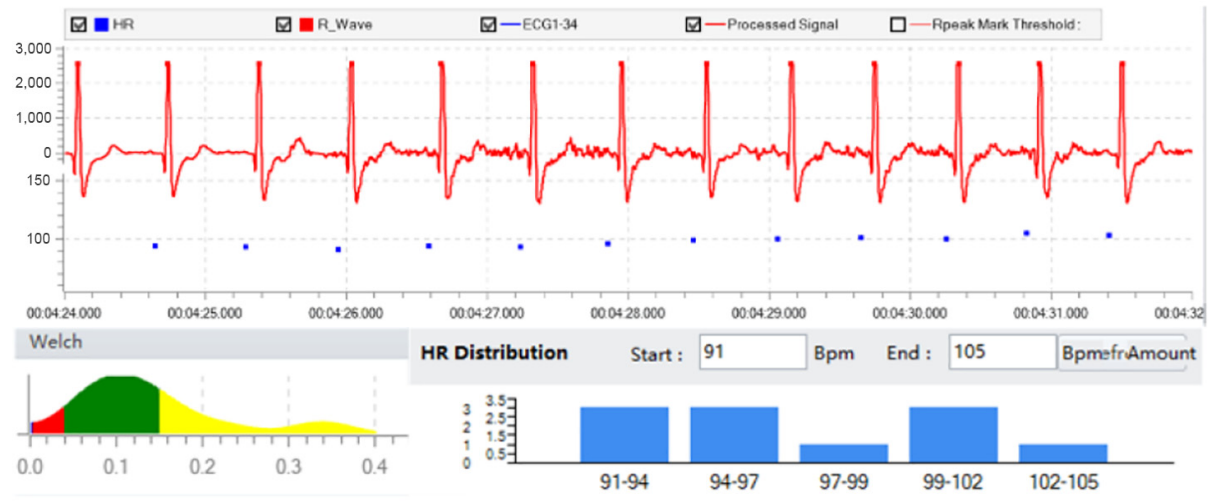

(b)

Notes: (a) Heart rate distribution and frequency spectrum distribution of the subjects in calm;

(b) Heart rate distribution and frequency spectrum distribution of the subjects in anxiety 
Table VI Statistics of driver's ECG characteristic data

\begin{tabular}{|c|c|c|c|c|}
\hline No. & $\mathrm{Em}$ & AVHR & $A V N N$ & SDNN \\
\hline \multirow[t]{15}{*}{1} & Calm & 84 & 710.79 & 30.97 \\
\hline & & $P N N_{50}$ & RMSSD & RWAVE \\
\hline & & 12.50 & 33.51 & 2557.98 \\
\hline & & TWAVE & $Q$ & $S$ \\
\hline & & 389.37 & 410.51 & 1491.03 \\
\hline & & UVLFIVLF & $L F / H F$ & $T P$ \\
\hline & & 0.10 & 1.20 & 384.74 \\
\hline & Anxiety & $A V H R$ & $A V N N$ & SDNN \\
\hline & & 95 & 632.60 & 129.36 \\
\hline & & $P N N_{50}$ & RMSSD & RWAVE \\
\hline & & 15.56 & 158.32 & 2559.27 \\
\hline & & TWAVE & $Q$ & $S$ \\
\hline & & 392.78 & 431.67 & 1554.49 \\
\hline & & UVLFIVLF & $L F / H F$ & $T P$ \\
\hline & & 0.07 & 1.07 & 2641.22 \\
\hline \multirow[t]{15}{*}{2} & Calm & 81 & 747.2 & 49.28 \\
\hline & & $P N N_{50}$ & RMSSD & RWAVE \\
\hline & & 23.68 & 39.99 & 2557.62 \\
\hline & & TWAVE & $Q$ & $S$ \\
\hline & & 378.68 & 430.65 & 1476.01 \\
\hline & & UVLFIVLF & $L F / H F$ & $T P$ \\
\hline & & 0.00 & 2.77 & 661.35 \\
\hline & Anxiety & $A V H R$ & AVNN & SDNN \\
\hline & & 91 & 657.96 & 57.79 \\
\hline & & $P N N_{50}$ & RMSSD & RWAVE \\
\hline & & 11.90 & 32.10 & 2559.17 \\
\hline & Anxiety & TWAVE & $Q$ & $S$ \\
\hline & & 363.74 & 469.37 & 1513.37 \\
\hline & & UVLFIVLF & $L F / H F$ & $T P$ \\
\hline & & 0.13 & 9.56 & 1123.26 \\
\hline \multirow[t]{15}{*}{3} & Calm & 85 & 702.33 & 72.57 \\
\hline & & $P N N_{50}$ & RMSSD & RWAVE \\
\hline & & 19.51 & 38.31 & 2126.88 \\
\hline & & TWAVE & $Q$ & $S$ \\
\hline & & 202.43 & 336.77 & 1250.24 \\
\hline & & UVLFIVLF & $L F / H F$ & $T P$ \\
\hline & & 0.01 & 25.53 & 3123.67 \\
\hline & Anxiety & $A V H R$ & AVNN & SDNN \\
\hline & & 102 & 586.65 & 45.39 \\
\hline & & $P N N_{50}$ & RMSSD & RWAVE \\
\hline & & 0 & 12.91 & 2234.62 \\
\hline & & TWAVE & $Q$ & $S$ \\
\hline & & 360.99 & 399.78 & 1260.28 \\
\hline & & UVLFIVLF & $L F / H F$ & $T P$ \\
\hline & & 0 & 12.89 & 749.11 \\
\hline$\ldots$ & & $\ldots$ & & $\ldots$ \\
\hline \multirow[t]{9}{*}{$n-1$} & Calm & 87 & 695.05 & 51.49 \\
\hline & & $P N N_{50}$ & RMSSD & RWAVE \\
\hline & & 44.44 & 51.56 & 1135.62 \\
\hline & & TWAVE & $Q$ & $S$ \\
\hline & & 188.98 & 192.67 & 672.91 \\
\hline & & UVLFIVLF & $L F / H F$ & $T P$ \\
\hline & & 0.03 & 24.76 & 1809.02 \\
\hline & Anxiety & AVHR & AVNN & SDNN \\
\hline & & 94 & 644.37 & $\begin{array}{l}31.37 \\
\text { ntinued) }\end{array}$ \\
\hline
\end{tabular}




\begin{tabular}{|c|c|c|c|c|}
\hline No. & $\mathrm{Em}$ & AVHR & $A V N N$ & $S D N N$ \\
\hline & & $P N N_{50}$ & RMSSD & RWAVE \\
\hline & & 2.5 & 27.41 & 1171.33 \\
\hline & & TWAVE & $Q$ & $S$ \\
\hline & & 110.52 & 209.44 & 685.18 \\
\hline & & UVLFIVLF & $L F / H F$ & $T P$ \\
\hline & & 0.01 & 7.18 & 565.47 \\
\hline \multirow[t]{15}{*}{$\mathrm{n}$} & Calm & 86 & 683.74 & 59.82 \\
\hline & & $P N N_{50}$ & RMSSD & RWAVE \\
\hline & & 33.33 & 46.88 & 1832.40 \\
\hline & & TWAVE & $Q$ & $S$ \\
\hline & & 280.73 & 210.32 & 850.72 \\
\hline & & UVLFIVLF & $L F / H F$ & $T P$ \\
\hline & & 0 & 5.44 & 1607.32 \\
\hline & Anxiety & $A V H R$ & AVNN & SDNN \\
\hline & & 91 & 643.24 & 161.12 \\
\hline & & $P N N_{50}$ & RMSSD & RWAVE \\
\hline & & 47.37 & 232.41 & 1920.80 \\
\hline & & TWAVE & $Q$ & $S$ \\
\hline & & 255.69 & 235.46 & 880.28 \\
\hline & & UVLFIVLF & $L F / H F$ & $T P$ \\
\hline & & 0.06 & 1.73 & 5497.79 \\
\hline
\end{tabular}

The real driving experiments in anxiety were carried out during morning peak hours of 7:00-9:00 and evening peak hours of 17:00-19:00 from Monday to Friday. The experimental process is shown in Figure 9.

Because driving simulation was used as a complement to real vehicle experiment, the simulation scenes were built according to the real road conditions, weather, traffic flow and other information of the real experiment. Thus, the experimental processes for the real driving and driving simulation are generally similar. Specifically, after that drivers' calm state is successfully induced, the driving experiment starts. During the whole process, soft music, bright light and fruit scents are provided to maintain drivers' calm state. After that drivers' anxious state is successfully induced, the driving experiment starts. During the whole process, loud music, subdued light and irritating odors are provided to keep or increase drivers' anxiety level. While drivers are also asked to finish some difficult tasks such as mathematical calculation or overtaking during driving.

\subsection{Assessing the level of the induced anxiety}

It is necessary to assess if subjects' anxiety is induced to a certain level of arousal, because too little or too much arousal can adversely affect subjects' performance in experiments. During the driving experiments, the facial expression, action, road conditions, driving speed and pedal strength were recorded in real time with the video monitoring system, speedometer and pedal dynamics instrument.

Subjects were asked to describe their self-perception of emotion in the conversation with experimenters. After the driving experiment, each subject was asked to watch the video immediately and report her emotional experience. The time segments of subjects' anxiety during driving were determined through the Beck Anxiety Inventory (BAI), self-perception, facial expression and behavioral action. The time segments of subjects' calm during driving were determined through the self-perception reporting method. The driver's ECG signal data in the selected time segments were used for the subsequent processing and analysis. In this study, the anxiety induction procedure is regarded as successful, if subjects have a score of 26 points or more for anxiety symptoms, as shown in Table III.

\subsection{Electrocardiograph signal data collection and preprocessing}

2.5.1 The principle of wavelet threshold denoising

The signal composition is:

$$
y_{t}=s_{t}+n_{t}
$$

where, $y_{t}$ represents a signal with noise, $s_{t}$ stands for the real signal, $n_{t}$ stands for the noise signal.

The basic principle of wavelet threshold denoising is that the signal loses correlation after wavelet transform, and the noise is distributed over the whole wavelet domain. In the wavelet domain, the signal coefficients are selected. It can be considered that the amplitude is smaller for the noise wavelet coefficients than the signal wavelet coefficients. Therefore, a threshold can be set. The wavelet coefficients higher than the threshold are kept, and the ones lower than the threshold are removed. That is, the noise wavelet coefficients are reduced to zero.

In this paper, the threshold is selected by:

$$
\lambda=\delta_{n} \sqrt{2 \log (N)}
$$

where, $\delta_{n}$ represents the standard variance of noise, and $N$ represents the length of the signal. 
The basic steps of wavelet denoising are:

- The wavelet basis and decomposition layer are chosen to calculate the wavelet decomposition coefficients.

- The appropriate threshold is chosen to denoise the high frequency coefficients.

- The low-frequency coefficients and the denoised highfrequency coefficients are reconstructed by wavelet transform, to obtain the denoised signal.

Figure 13 Subjects' ECG characteristic indicators in calm and anxiety

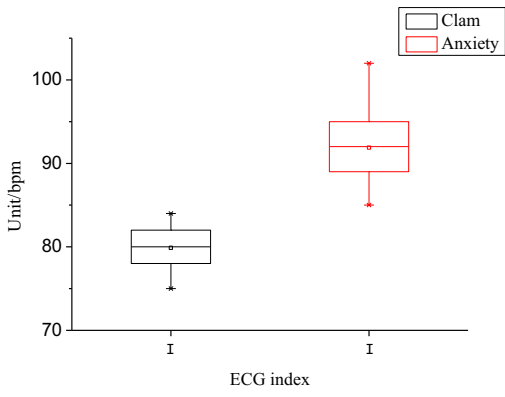

(a)

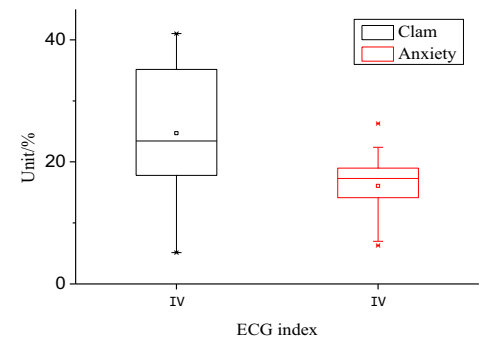

(c)

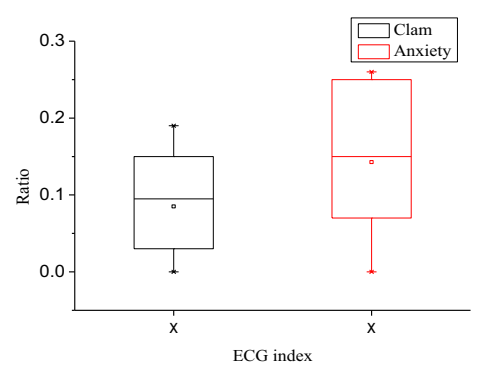

(e)

\subsubsection{Electrocardiograph signal data preprocessing}

During the data collection, there are unavoidable noise in the original ECG signals due to the factors of experimental environment, equipment, and human. Three main types of noise are shown as follows:

1 Motion artifact: the step change in ECG signal is generated for the dislocation movement between electrodes and skin.

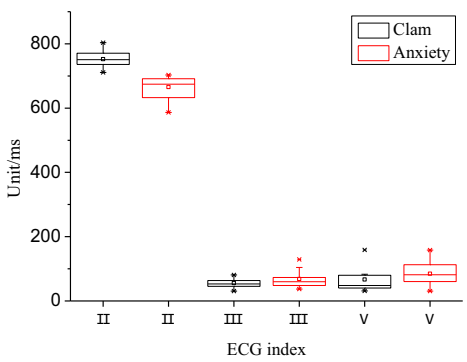

(b)

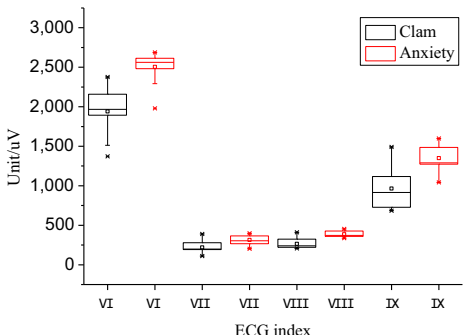

(d)

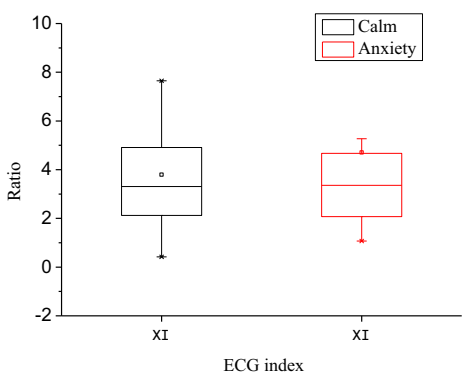

(f)

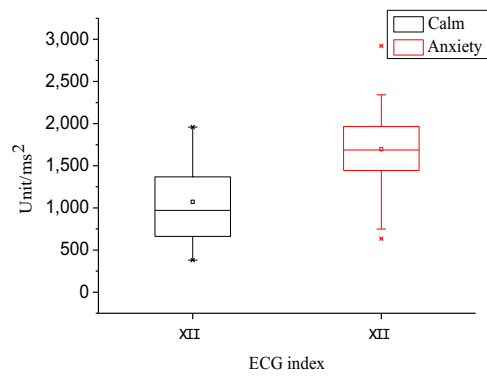

(g)

Notes: (a) I- $A V H R$; (b) II- $A V N N$, III-SDNN, V-RMSSD; (c) IV-PNN ;

(d) VI-RWAVE, VII-TWAVE, VIII- $Q$, IX-S; (e) X-UVLF/VLF; (f) XI- $L F / H F$;

(g) XII-TP 
2 Power frequency interference: the noise is generated for the electromagnetic radiation in experimental environment.

3 Sensor internal interference: the noise is generated for the current passing through the sensor's internal components.

Before analyzing data, it is an important first step to remove noise from the raw data. The PSYLAB software was used to preprocess the raw ECG signal data, as shown in Figure 10. The definitions of the parameters for denoising preprocess are shown in Table IV. The noise of motion artifact, power frequency interference and sensor internal interference can be removed through noise-suppressed processing to an acceptable level. The comparison of ECG signal before and after denoising is shown in Figure 11.

\subsubsection{Electrocardiograph signal data collection}

Each subject was involved in two real driving experiments and three driving simulation driving experiments, in which they were induced to feel either calm or anxious. A total of 1327 groups of effective data were obtained. The variables and symbols for ECG signals are shown in Table V. Parts of the experimental data are shown in Figure 12 and Table VI.

\section{Results and discussion}

To identify the differences of driver's ECG characteristics in calm and anxious states, subjects' ECG characteristic indicators in the two emotions were compared. In Figure 13, the maximum, minimum, median, upper and lower quartiles of each ECG characteristic indicator of the two emotions are demonstrated.

In Figure 13, it was observed that for $A V H R$ and $A V N N$, there is an obvious difference between anxious and calm states in mean and range. The $A V H R$ difference presents that anxiety has a higher level and greater variability in $A V H R$ than calm. The $A V N N$ difference shows that anxiety has a lower level and more variable distribution in $A V N N$ than calm. For $A V N N$, the boxplot is symmetric for calm and skewed for anxiety, showing that the calm data follows a normal distribution and most of the anxiety data are in the top. For $P N N_{50}$, the lengths of both the box and the whiskers are greater in calmness than in anxiety. This shows that the data set of $P N N_{50}$ is more dispersed in calmness than in anxiety. These evidences suggest that female drivers have a more rapid heart rate and a shorter heartbeat interval in anxiety than in calmness. Anxious Female drivers experience heartbeat fluctuation in high frequency, and the amplitudes of these fluctuations are prone to be consistent. The differences between successive $\mathrm{NN}$ intervals are less likely to be greater than $50 \mathrm{~ms}$. The cases in clam are the opposite. Moreover, it was noticed that there are no obvious differences in the ECG characteristic indicators between anxiety and calmness, including SDNN, UVLF/VLF, and TP.

Statistical analysis was performed using SPSS Statistics 23.0 where the confidence interval was set at 95 per cent. The paired T-test was used to determine whether there are differences between the two emotions in the ECG characteristic indicators, and the results are shown in Table VII.

The results show that there is a significant difference between calm and anxious states in the seven ECG indicators, including $A V H R, A V N N, P N N_{50}, R W A V E$, $R M S S D$, Qand $S(p<0.05)$. There is no significant difference between calm and anxious states in the other indicators. The results in Figure 13 and Table V show that the average heartbeat and the percent of adjoin heart beat are greater in calm than in anxious state. The average heart rate, the average value of adjoin RR intervals, the $\mathrm{R}$ wave peaks, $\mathrm{Q}-$ point and S-point peaks of the QRS wave are lower in calm than in anxious state. The results indicate that compared with calm state, female drivers have a faster heart rate, a shorter heartbeat interval, a more frequent heartbeat fluctuation, a longer conduction time of the heart chamber, and a more obvious manifestation of myocardial ischemia in anxious state. These phenomena suggest that woman drivers in anxious state may spend a shorter time for depolarization and repolarization and present with much larger QRS amplitudes than ones in the calm state.

Table VII Paired T-test results of driver's ECG characteristics in calm and anxiety

\begin{tabular}{|c|c|c|c|c|c|c|c|c|c|}
\hline & & Mean & SD & $\begin{array}{l}\text { ed samples } \\
95 \% \text { Conf } \\
\text { of the } \\
\text { Std. Error } \\
\text { Mean }\end{array}$ & $\begin{array}{l}\text { ce interval } \\
\text { erence } \\
\text { Lower }\end{array}$ & Upper & $t$ & df & $\begin{array}{c}\text { Sig. } \\
\text { (two-tailed) }\end{array}$ \\
\hline$\overline{\text { AVHR }}$ & Calm-Anxiety & -8.778 & 3.541 & 0.835 & -10.538 & -7.017 & -10.518 & 17 & $0.000^{*}$ \\
\hline AVNN & Calm-Anxiety & 78.191 & 27.373 & 6.452 & 64.579 & 91.834 & 12.119 & 17 & $0.000^{*}$ \\
\hline SDNN & Calm-Anxiety & -12.250 & 43.684 & 10.296 & -33.979 & 9.468 & -1.190 & 17 & 0.250 \\
\hline $\mathrm{PNN}_{50}$ & Calm-Anxiety & 12.660 & 12.342 & 2.909 & 6.523 & 18.797 & 4.352 & 17 & $0.037^{*}$ \\
\hline RMSSD & Calm-Anxiety & -81.410 & 67.382 & 15.882 & -114.924 & -47.907 & -5.126 & 17 & $0.041^{*}$ \\
\hline RWAVE & Calm-Anxiety & -53.320 & 43.266 & 10.198 & -74.843 & -31.812 & -5.229 & 17 & $0.000^{*}$ \\
\hline TWAVE & Calm-Anxiety & -12.110 & 46.602 & 10.984 & -35.29040 & 11.059 & -1.103 & 17 & 0.285 \\
\hline $\mathbf{Q}$ & Calm-Anxiety & -19.556 & 16.940 & 3.993 & 11.132 & 27.98 & 4.898 & 17 & $0.000^{*}$ \\
\hline$S$ & Calm-Anxiety & -33.059 & 19.197 & 4.525 & 23.513 & 42.606 & 7.036 & 17 & $0.000^{*}$ \\
\hline UVLF/VLF & Calm-Anxiety & 0.005 & 0.084 & 0.198 & -0.037 & 0.047 & 0.253 & 17 & 0.803 \\
\hline LF/HF & Calm-Anxiety & -1.002 & 8.436 & 1.988 & -5.197 & 3.193 & -0.504 & 17 & 0.621 \\
\hline TP & Calm-Anxiety & -111.10 & 1231.807 & 290.340 & -723.693 & 501.433 & -0.383 & 17 & 0.707 \\
\hline
\end{tabular}




\section{Conclusion}

In this paper, the real driving experiments and driving simulations were designed and conducted to collect driver ECG signal data. It was found that there is significant difference between calmness and anxiety on the time domain, frequency domain and waveform of the ECG characteristic indicators, including $A V H R, A V N N, P N N_{50}, R W A V E$, $R M S S D, Q$ and $S$. Compared with the calm state, female drivers have a faster heart rate, a shorter heartbeat interval, a more frequent heartbeat fluctuation, a longer conduction time of the heart chamber, and a more obvious manifestation of myocardial in anxiety.

Our findings of this study suggest that ECG signals can be used to identify driver's anxious and calm states during driving. It can be used for development of driving warning system, which can predict the unsafe driving behavior caused by anxiety emotion and then send alarm signals to drivers. Moreover, our findings can also be used to develop the personalized driving warning system for different types of drivers. This study would be of great theoretical significance and application value for the development of the intelligent and connected vehicles. Further studies are required to confirm the above findings with more number of subjects and by collecting more data from each subject to improve the accuracy and reliability of the experiments.

\section{References}

Asher, M., Asnaani, A. and Aderka, I.M. (2017), "Gender differences in social anxiety disorder: a review", Clinical Psychology Review, Vol. 56 No. 7, pp. 1-12.

Ba, Y., Zhang, W. and Chan, A.H. (2016), "How drivers fail to avoid crashes: a risk-homeostasis/perception-response (RH/ PR) framework evidenced by visual percepyion, electrodermal activity and behavioral", Transportation Research Part F: Traffic Psychology and Behaviour, Vol. 43, pp. 24-35.

Barnard, M.P. and Chapman, P. (2018), "The effects of instruction and environmental demand on state anxiety, driving performance and autonomic activity: are egothreatening manipulations effective", Transportation Research Part F: Traffic Psychology and Behaviour, Vol. 55, pp. 123-135.

Cestac, J., Paran, F. and Delhomme, P. (2011), "Young drivers' sensation seeking, subjective norms, and perceived behavioral control and their roles in predicting speeding intention: how risk-taking motivations evolve with gender and driving experience", Safety Science, Vol. 49 No. 3, pp. 424-432.

Chen, H., Rau, P. and Guo, Z. (2017), "The effects of V2V information on visual attention and driving performance of the drivers", Chinese fournal of Ergonomics, Vol. 23 No. 4, pp. 44-47.

Chen, Y.L., Chang, C.L. and Yeh, C.S. (2017), "Emotion classification of YouTube videos", Decision Support Systems, Vol. 101, pp. 40-50.

Clingingsmith, D. (2016), "Negative emotions, income, and welfare: causal estimates from the PSID", fournal of Economic Behavior E Organization, Vol. 130, pp. 1-19.

Dubovski, N., Ert, E. and Niv, M.Y. (2017), "Bitter mouthrinse affects emotions", Food Quality and Preference, Vol. 60, pp. 154-164.

Fernández, B. (2016), "Psychophysiological, subjective and behavioral differences between high and low anger drivers in a simulation task", Transportation Research Part F: Traffic Psychology and Behavior, Vol. 42 No. 2, pp. 365-375.

Ford, B.Q. and Mauss, I.B. (2015), "Culture and emotion regulation”, Current Opinion in Psychology, Vol. 3, pp. 1-5.

Gallagher, C.E., Watt, M.C. and Weaver, A.D. (2017), "I fear, therefore, I shop!' Exploring anxiety sensitivity in relation to compulsive buying", Personality and Individual Differences, Vol. 104, pp. 37-42.

Geethanjali, B., Adalarasu, K. and Jagannath, M. (2018), "Music induced emotion using wavelet packet decomposition-An EEG study", Biomedical Signal Processing and Control, Vol. 45, pp. 115-128.

Goshvarpour, A. and Abbasi, A. (2017), "An accurate emotion recognition system using ECG and GSR signals and matching pursuit method", Biomedical fournal, Vol. 40 No. 6, pp. 355-368.

Handouzi, W., Maaoui, C. and Pruski, A. (2014), "Objective model assessment for short-term anxiety recognition from blood volume pulse signal", Biomedical Signal Processing and Control, Vol. 14, pp. 217-227.

Hosking, S.G., Liu, C.C. and Bayly, M. (2010), "The visual search patterns and hazard responses of experienced and inexperienced motorcycle riders", Accident Analysis $\mathcal{G}$ Prevention, Vol. 42 No. 1, pp. 196-202.

Jaeger, S.R., Lee, S.M. and Kim, K. (2017), "Measurement of product emotions using emoji surveys: case studies with tasted food and beverages", Food Quality and Preference, Vol. 62, pp. 46-59.

Jia, D. and Ngoduy, D. (2016), "Platoon based cooperative driving model with consideration of realistic inter-vehicle communication", Transportation Research Part C: Emerging Technologies, Vol. 68, pp. 245-264.

Jia, H.F., Si, Y.X. and Tang, M. (2006), "Study on driver information processing mode based on cognitive psychology", China Safety Science fournal, Vol. 16 No. 1, pp. 22-25.

Kang, G.E. and Gross, M.M. (2016), "The effect of emotion on movement smoothness during gait in healthy young adults", Fournal of Biomechanics, Vol. 49 No. 16, pp. 4022-4027.

Kirwan, M., Pickett, S.M. and Jarrett, N.L. (2017), "Emotion regulation as a moderator between anxiety symptoms and insomnia symptom severity", Psychiatry Research, Vol. 54, pp. 40-47.

Minhad, K.N., Mdali, S.H. and Ibne, M.B. (2017), "Happyanger emotions classifications from electrocardiogram signal for automobile driving safety and awareness", fournal of Transport \& Health, Vol. 7 No. 1, pp. 75-89.

Naveteur, J., Coeugnet, S. and Charron, C. (2013), "Impatience and time pressure: subjective reactions of drivers in situations forcing them to stop their car in the 
road", Transportation Research Part F: Traffic Psychology and Behaviour, Vol. 18 No. 22, pp. 58-71.

Panayiotou, G., Karekla, M. and Leonidou, C. (2017), "Coping through avoidance may explain gender disparities in anxiety", Fournal of Contextual Behavioral Science, Vol. 6 No. 2, pp. 215-220.

Reimer, B., Mehler, B. and Coughlin, J.F. (2011), "The impact of a naturalistic hands-free cellular phone task on heart rate and simulated driving performance in two age groups", Transportation Research Part F: Traffic Psychology and Behaviour, Vol. 14 No. 1, pp. 13-25.

Rymarcayk, K., Biele, C. and Grabowska, A. (2011), "EMG activity in response to static and dynamic facial expressions", International fournal of Psychophysiology, Vol. 79 No. 2, pp. 330-333.

Schmidt-Daffy, M. (2012), "Velocity versus safety: impact of goal conflict and task difficulty on drivers' behavior, feeling of anxiety, and electrodermal responses", Transportation Research Part F: Traffic Psychology and Behaviour, Vol. 15 No. 3, pp. 319-332.

Scott-Parker, B. (2017), "Emotions, behaviour, and the adolescent drive: a literature review", Transportation Research Part F: Traffic Psychology and Behaviour, Vol. 50, pp. 1-37.

Takahashi, R., Kobayashi, M. and Sasaki, T. (2017), "Driving simulation test for evaluating hazard perception: elderly driver response characteristics", Transportation Research Part F: Traffic Psychology and Behaviour, Vol. 49, pp. 257-270.

Velez, E.R., Leeuwen, P.M. and Happee, R. (2016), "The effects of time pressure on driver performaance and physiological activity: a driving simulator study", Transportation Research Part F: Traffic Psychology and Behaviour, Vol. 41, pp. 150-169.
Vingilis, E., Yenier, Z.Y. and Fischer, P. (2016), "Selfconcept as a risky driver: mediating the relationship between racing video games and on-road driving violations in a community based sample", Transportation Research Part F: Traffic Psychology and Behaviour, Vol. 43, pp. 15-23.

Wang, L., Bie, Y. and Li, S.W. (2016), "The impact of roadside landscape colors on driver's mean heart rate considering driving time", Transportation Research Part F: Traffic Psychology and Behaviour, Vol. 42 No. 1, pp. 151-161.

Wang, X.Y., Zhang, J.L. and Ban, X.G. (2013), Vehicle Driving Tendency Identification Based on Collaborative Simulation of Dynamic Human-Vehicle Environment, Science Press, Beijing.

Wilhelm, F.H., Rattel, J.A. and Wegerger, M. (2017), “Attend or defend? Sex differences in behavioral, autonomic, and respiratory response patterns to emotion-eliciting films", Biological Psychology, Vol. 130, pp. 30-40.

Yan, L.X., Wu, C.Z. and Gao, S. (2013), "Study on the relationship between driver's individual factors and driving anger", Fournal of Transport Information and Safety, Vol. 31 No. 6, pp. 119-124.

Zhang, M.H. (2014), Different Risk Levels Based on Real Vehicle Simulation Study on the Behavior of New Drivers following a Vehicle, Shanghai Jiaotong University, p. 33.

Zhang, X.B., Zuo, B., Erskine, K. and Hu, T. (2016), "Feeling light or dark? Emotions affect perception of brightness", Fournal of Environmental Psychology, Vol. 47, pp. 107-111.

\section{Corresponding author}

Xiaoyuan Wang can be contacted at: wangxiaoyuan@qust. edu.cn 